\title{
My Relations with Physicists Who Contributed Essentially to Our Understanding of Superconductivity
}

\author{
Zygmunt M. GalasiewiCZ
}

Retired Professor of Wrocław University

I mention here N.N. Bogolyubov, L.D. Landau, P.L. Kapitza, A.A. Abrikosov, V.L. Ginzburg, J. Bardeen, and K.A. Müller. I presented the essence of their results on the way to a better understanding the superconductivity (six of them have received the Nobel Prize). I have selected various interesting episodes from their biographies. I have supplemented all this with my personal observations from numerous, meetings with them.

I have been interested in theory of superconductivity since 1958, when I arrived at the Joint Institute of Nuclear Research (JINR) in Dubna. I arrived there in order to work under direction of the physicist and mathematician Professor Nikolai Nikolaevich Bogolyubov. At the time he was the Director of the Laboratory of Theoretical Physics JINR. My arrival in Dubna was a consequence of the fact that in 1956 I have received Ph.D. for the dissertation, which provided a broad generalization of the ideas of Prof N.N. Bogolyubov devoted to the many-body theory. Namely, he introduced the so-called supplementary variables, in order to describe in a more efficient way, the system of interacting electrons in metals. I generalized this idea and considered together electrons and crystal-lattice ions. I presented quite new approach to the theory of metallic bond in metals. My supervisor, Prof. R.S. Ingarden, when visiting Laboratory Kharkov (Ukraine), has learned about the Bogolyubov ideas and copied by hand the Bogolyubov paper containing about 60 pages. During the period of preparations of my thesis I studied other Bogolyubov papers and presented them at a seminar in our Theoretical Physics Department.

Nikolai Nikolaevich Bogolyubov (1909-1992), Ukrainian, attended the school in Kiev where he lived with a Polish family. Because of this situation he understood Polish and liked Polish people. Knowing my interest in the Bogolyubov work, Prof. Rzewuski took steps towards organizing my long-term visit to Dubna after the end of his stay there. Bogolyubov's father was an orthodox-rite priest and later, professor of theology. When Nikolai Nikolaevich was 13 years old he already attended in Kiev seminars in the Department of Mathematical Physics, directed then by well known mathematician Prof. N.N. Krylov. His first scientific paper, with Krylov, was published in 1925, i.e. at the age of 15 . As the son of a priest (who was arrested without any accusation and died very soon later), he could not study at "Soviet" Universities. Fortunately, he worked scientifically and in 1930 received his Ph.D. in Mathematics (at the age of 21). As we see, he had no formal university studies. Since 1965, N.N. Bogolyubov was the Director of JINR. At 
that time, there was a joke circulating in Dubna. Namely, upon suggestions from the Moscow Authority of the Institute, it was decided to evaluate the educational level of the Institute employees. The splendid statistics has been spoiled by janitors and... by Nicolai N. Bogolyubov.

Bogolyubov became interested in the theory of superconductivity only in 1957, when J. Bardeen, L. Cooper, and J. Schrieffer (BCS) published the fundamental paper explaining the essence of the phenomenon. Earlier, in 1950, H. Fröhlich proved that in metals the interaction of electrons with oscillations of the crystal lattice (formed by positively charged ions ) leads in consequence to the effective attraction among electrons. Therefore, the influence of the "environment" leads to the effective attraction instead of the Coulomb repulsion. The authors of the BCS theory were influenced by the earlier paper by L.N. Cooper (1956). He considered the system of $N+2$ fermions. The $N$ of them are non-interacting particles, but because of the Pauli principle they occupy all momenta states in Fermi sphere up to $p_{\mathrm{F}}$. The two remaining electrons located close to Fermi surface $\left(p \sim p_{\mathrm{F}}\right.$, but $p>p_{\mathrm{F}}$ ) interact via an attractive force. In consequence, a bound state of two fermions is formed, i.e. the state with negative eigenenergy $E$. The creation of the electrons bound state - named the Cooper pair - leads to a stable configuration, because now the energy of the $(N+2)$ fermions is smaller than that of $(N+2)$ non-interacting fermions. Bardeen, Cooper, and Schrieffer (1957) proved that the essence of superconductivity are correlated electron pairs. The partners of the pair have both the opposite momenta and spins: $(\boldsymbol{p}, 1 / 2),(-\boldsymbol{p},-1 / 2)$, where $p \sim p_{\mathrm{F}}$. The energy of the mentioned correlation is expressed by the so-called energy gap $\Delta$, which appears in the expression for the energy spectrum $E_{\boldsymbol{p}}=2 \sqrt{\Delta^{2}+\left(\varepsilon_{\boldsymbol{p}}-\varepsilon_{\mathrm{F}}\right)^{2}}$ of superconducting system.

Till 1958 Bogolyubov was interested in the theory of superfluidity based on the idea of the Bose-Einstein condensation. In 1947 he published a really very important paper On the Theory of Superfluidity. It is one of unique examples of exactly solvable models. In this case, the system is that of weakly interacting bosons. In order to diagonalize the Hamiltonian of this model Bogolyubov proposed, now very well known, the canonical transformation, $\{u, v\}$ describing a change to new boson annihilation and creation operators. The Hamiltonian expressed in terms of these new operators has been diagonalized thanks to the exact solution of two equations for the coefficients $u, v$ of the mentioned Bogolyubov transformation. One of these equations guarantees that the new operators will obey the same boson commutation relations. The second equation is the condition that the term of the transformed Hamiltonian, which would lead to infinities, should be equal to zero. This second equation was named by Bogolyubov the equation of compensation of dangerous diagrams. In the Bogolyubov paper there was also result important for the phenomenological Landau theory (1941) of the superfluid helium 4. Namely, Bogolyubov showed that the energy spectrum $\varepsilon=\varepsilon(p)$ has at the beginning (i.e. for $p \sim 0$ ) only one branch, the phonon branch, linear in $p, \varepsilon_{\mathrm{ph}}=c p$, where $c$ is the sound velocity. This result turned out to be very important and will be discussed later on. Evident analogies between superfluidity and superconductivity inspired Bogolyubov to apply the transformation of the type of $\{u, v\}$ to the system of fermions (electrons). For the Coulomb repulsion the equation of compensation of dangerous diagrams leads to "nonphysical" solutions. The quite formal change of the interaction sign (to attraction) leads to "physical solutions". But at that time the consideration of electrons attracting each other was absolutely "nonphysical". 
After a period of interest in many body problems (statistical physics) Bogolyubov was deeply involved in the problems of field theory. Therefore unfortunately, he did not pay any attention to the paper by Fröhlich. The attraction was the basis of the BCS theory. The paper of Bardeen, Cooper, and Schrieffer (1957) caught the Bogolyubov attention to the effective attraction. He immediately published papers important to the essence of superconductivity: in 1957 - Dubna preprint and in 1958 Nuovo Cimento article About New Method in the Theory of Superconductivity. This new method was really the application of the "old" $\{u, v\}$ canonical transformation (1947) to the "old" Fröhlich Hamiltonian (1950). The successful Bogolyubov approach to the theory of superconductivity turned out to be much more efficient than the BCS one.

When I arrived in Dubna in 1958, Bogolyubov was just interested in collective excitations in superconductors and their influence on electrodynamics of the superconducting state. He showed that in the case of external magnetic field (having his "own" momentum $\boldsymbol{q}$ ), the previous canonical transformation $\{u, v\}$ should be generalized. Namely, the "old" transformation joined only the diagrams connected with momentum $\boldsymbol{p}$ with diagrams connected with momentum $-\boldsymbol{p}$, so that $\boldsymbol{p}_{1}+\boldsymbol{p}_{2}=\mathbf{0}$. In the case of electromagnetic field the transformation should be generalized, taking now into account relation $\boldsymbol{p}_{1}+\boldsymbol{p}_{2}=\boldsymbol{q}$.

Professor Bogolyubov proposed me considering the electrodynamics of superconducting state on the basis of the called method of approximate second quantization, which he elaborated earlier in his book (in Ukrainian) devoted to statistical physics. The generalized canonical transformation led to very general form of Hamiltonian. On his basis one can consider, among others, simultaneously collective oscillations of the pairs of fermions with opposite spins (total spin of the pair equal to 0 , so-called "s" pairing) and the pairs with parallel spins (total spin of the pair +1 or -1 , the so-called "p" pairing). The equation of compensation of the dangerous diagrams was traditionally connected with "s" pairs. I got new idea to write up quite formally the equation of compensation of dangerous diagrams for "p" pairs. In this manner I described the properties of new anisotropic phase of the system of fermions. The results were first published as the JINR Dubna preprint (1959). This was, fortunately, the first theory of anisotropic superfluid phase with "p" pairs of fermions, called now the polar phase.

In 1972 the superfluidity of helium 3 was discovered. It is the Fermi fluid like a system of electrons in metals (helium 3 atoms have spin $1 / 2$ ), only electrical neutral. Superfluidity denotes here the flow without viscosity and corresponds to the Ohm resistance in the case of electrons. Later, a more careful experimental analysis of the superfluidity in helium 3 showed that it is connected with the anisotropic phase with "p" pairs. In 1975 A. Leggett published in Rev. Mod. Phys. a review paper, in which he described theoretically all anisotropic phases (based only on "p" pairs). This paper was the basis of the Nobel Prize awarded to A. Leggett in 2003. He quoted my paper edited in Dubna in 1959 (and published in Acta Phys. Pol. in 1960). In connection with this N.N. Bogolyubov, now the Director of JINR, decided in 1975 to reedit my two Dubna preprints and in the Introduction he underlined that the theory of the Fermi systems with "p" pairing was first proposed in the Dubna. This theory formed a basis of my habilitation in 1961. From 1960, for many years, I was the member of the Scientific Council in the Laboratory of Theoretical Physics of JINR. I attended regularly (twice per year) the meeting of the Council. Moreover, on the occasion of Nikolai Nikolaevich birthday in August, there were organized Conferences devoted to the problems of statistical physics. I participated in these meetings. Therefore, after my longer permanent stay in Dubna 
(1958-1959) I visited JINR several times per year. Bogolyubov always found time to invite me for longer discussions not only the scientific.

I was really considered as close connected to Bogolyubov. Because of this I was mentioned in two books. The first one has been published in Kiev (1975). Soniatna teorema (Sunny Theorem), biographic novel about N.N. Bogolyubov written by Bogdan Weres. The second novel was published in Warsaw by Włodzimierz Kusz: Too near Neighborhood - The Scenes of the Soviet-Polish Atomic Physics after the II World War. On the pages 52-55 I am presented as Dr. Aleksander W. from Wrocław, closely connected with Bogolyubov.

I collaborated with JINR in Dubna for 41 years (1958-1999). In 2006 there were celebrations of the 50-years jubilee of JINR. According to this occasion I was awarded the Medal 50 Years of the Participation of Poland in JINR. Results of my investigations presented in scientific papers and at international conferences attracted attention of specialists from superconductivity and superfluidity and often I was looked upon as a Bogolyubov pupil. For this reason I was awarded by Professor D. ter Haar with order to write two books for the Pergamon Press in Oxford: Superconductivity and Quantum Fluids (1970) and Helium 4 (1971).

The essential distinction was for me the Maria Skłodowska-Curie Award (1983): for the series of papers presenting outstanding results in the theory of quantum fluids which I have received with Prof. Jerzy Czerwonko.

It is worth stressing that thanks to the long-term visits of the younger members from our Wrocław Institute in the Laboratory of Theoretical Physics, directed by N.N. Bogolyubov, there were prepared 4 doctorates and 6 habilitations. In order to underline and honor the merits of Nikolai Nikolaevich in the scientific exchange with our University, the Senate awarded him with the degree of doctor Honoris Causa (1969). Promotion took place in 1972. During his visit in Wrocław Bogolyubov was guided, among others, to several historical churches. It occurred that his knowledge about many Bible scenes exceeded the knowledge of his guides. In reality, he was the guide. It was also for me very pleasant to know that in 1987 the International Center for Theoretical Physics in Trieste established the Bogolyubov Prize in Mathematics and Solid State Physics for scientists from developing countries.

The scientists foreigners working in JINR had a privilege i.e. permanent permit to travel to Moscow $(150 \mathrm{~km})$. In the USSR the foreigners could, in general, move from the place of register only up to $40 \mathrm{~km}$ away. I used regularly the possibility to visit Moscow to attend almost every weak seminars in the Division of Theoretical Physics of the Institute of Physical Problems. The leader of these Seminars was famous theoretician Professor Lev Davidovich Landau. Among participants there were prominent as P. Kapitza, A. Abrikosov, W. Ginzburg, the future, like L.D. Landau, Nobel Prize winners. I find that it is interesting to describe the complicated relations N.N. Bogolyubov - L.D. Landau.

L.D. Landau elaborated the theory of superfluid helium 4 impressed, among others, by exciting experiments of P. Kapitza. In the extensive paper (1941) he derived (Chapter 7) the famous hydrodynamic equations for superfluid helium 4. Chapter 2 is devoted to the energy spectrum of this bosonic quantum fluid. Landau assumed a priori (!) that the relation $\varepsilon=\varepsilon(p)$ has two branches, both from the point $p=0$. Namely, the "phonon" $\varepsilon_{\mathrm{ph}}$ branch and the "roton" $\varepsilon_{\text {rot }}$ branch: $\varepsilon_{\mathrm{ph}}(p)=c p, \varepsilon_{\mathrm{rot}}(p)=\Delta+p^{2} / 2 \mu$, which for $p=0 \rightarrow \varepsilon_{\mathrm{ph}}(0)=0, \varepsilon_{\mathrm{rot}}(0)=\Delta$, and $\mu$ denotes the effective mass of roton. It was however signalled that something is wrong. Namely, known experimental physicist 
V. Peshkov performed calculations based on the form of the energy spectrum proposed by Landau and got results different from experimental ones. As I mentioned, N.N. Bogolyubov in his (1947) paper proved exactly that in vicinity of $p=0$ energy spectrum has only the phonon branch $\varepsilon(p)=\varepsilon_{\mathrm{ph}}(p)=c p$. Bogolyubov presented his paper at the Seminar of the Academy of Sciences in Moscow and published it in J. Phys. (USSR) 11 p. 23(!) (1947). L.D. Landau was present at Bogolyubov's lecture. Shortly after mentioned lecture Landau sent the paper, published also in J. Phys. (USSR) 11, p. 91(!) (1947) and devoted to the energy spectrum of superfluid helium 4 (so, his paper was placed 70 pages after that of Bogolyubov). Landau assumed now that in the vicinity of $p=0$ the energy spectrum of Bose quantum fluid has only phonon branch $\varepsilon(p)=c p$, as follows from the Bogolyubov paper. For greater $p$ the spectrum $\varepsilon(p)$ reaches some maximum and then falls to the so-called roton-minimum having value $\Delta$ and reached at $p=p_{0}$. In the vicinity of $p_{0}$ Landau therefore assumed then the energy spectrum $\varepsilon_{\text {rot }}(p)=\Delta+\left(p-p_{0}\right)^{2} / 2 \mu$, with $\varepsilon_{\text {rot }}\left(p_{0}\right)=\Delta$. In the new paper of Landau the energy spectrum for superfluid helium 4 has been assumed correctly and the theoretical predictions based on it was in agreement with experimental data. For the mentioned two papers L.D. Landau received in 1962 the Nobel Prize. Unfortunately, what was unpleasant, that in his paper (1947) he did not quote the Bogolyubov paper On the Theory of Superfluidity (1947), which, as we know, was presented earlier than his paper. In consequence, relations between these two scientists were only coldly correct. As mentioned, Dubna was opened in 1956. In order to create the international recognition to its Scientific Council were nominated most famous Russian (Soviet) physicists. Among them also L.D. Landau. But he never accepted the invitation to the Council meetings or to serious scientific conferences organized in Dubna. One can explain this situation only by not good relations with Bogolyubov. Unexpectedly, the relations changed in the positive direction. Namely, Bogolyubov had successes in the development of the theory of superconductivity. The domestic Academy of Sciences edited his book New Method in the Theory of Superconductivity. N.N. Bogolyubov was awarded by the highest Scientific Prize in USSR. During the celebration L.D. Landau publicly delivered warm congratulations. Since this time the relations between them were much better. Finally, in January 1962 Landau accepted Bogolyubov invitation to the meeting of Scientific Council of JINR. Landau traveled to Dubna by car. Just during that trip the known tragic car collision took place. The great truck stroked the car backwards. As I mentioned, just in 1962 he got Nobel Prize for the equation for superfluid helium 4 (helium II).

L.D. Landau (22.I.1908-01.IV.1968) was born in Baku. In 1927 he finished studies at the University in Leningrad. In 1929 he got for 1.5 year the "Soviet" scholarship to visit scientific institutions in Germany, Denmark, Switzerland, Holland, and England. The most important for him was the stay in Copenhagen. He gained high appreciation by Niels Bohr, who helped him to get extra (high) Rockefeller Scholarship. Landau could visit e.g. W. Pauli in Zürich, and E. Rutherford in Cambridge. There he met P. Kapitza. During his absence his father, engineer and petrol specialist in Baku, was sent to a concentration camp for ten years.

When Landau returned from abroad, Kapitza helped him to avoid K.G.B. (USSR secret police). Namely, if somebody after a stay in capitalistic country was again in socialistic "paradise", he felt unhappy looking at empty shops, reading controlled newspapers, etc. Therefore, such people were directed for 6-12 months to special camps, where they worked. Finally, when they returned back home, they became again very happy. 
Peter L. Kapitza (9.VII.1894-8.IV.1984), the son of general at tsarist time, was born in Kronstadt. In 1918 he finished studies at the Technical University in Petersburg. In 1921 he left Russia for Cambridge, where he worked at the Cavendish Laboratory, under direction of Ernest Rutherford. Kapitza was considered by Rutherford as a very good specialist and was in the period 1924-1932 the deputy director of this Laboratory. He was very active and spontaneous in scientific and private activity. Cambridge citizens learned about them, when he drove a car with an unlimited speed, or quite naked took bath in the neighboring river. At the same time he was a giant in scientific work. Therefore, Rutherford built for Kapitza a separate, very well equipped, Laboratory founded by American multimillionaire Mond. For this reason it was named Mond Laboratory and opened in January 1933. Unfortunately, Stalin decided that Kapitza should work for USSR and not for the Capitalists. Therefore, Kapitza was invited in 1934 to Moscow for a celebration in Academy of Sciences. And he could not return back to England. Since, even very strong protests did not help, Rutherford decided present to Kapitza all equipment of the Mond Laboratory. This equipment was transported from England by a special ship. The head of this enterprise was P.A.M. Dirac.

I met Dirac several times (e.g. in Trieste). He recollected cheerfully about his experiences in a communistic country. As a compensate for the capture, "good" Stalin ordered to build for Kapitza a private residence in Moscow in the old English style. In addition, in the vicinity a Workshop was constructed, quite private Laboratory for Kapitza. The greatest building near (in "socialistic" style) was for the Institute of Physical Problems, where Kapitza was the director in the periods 1935-46 and 1955-84. The period 1946-1955 is the time of the home arrest in his "dacha" (summer house) close to Moscow. Namely, Kapitza was nominated as a member of the USSR Special Committee to prepare a production of atomic bombs. The head of the project was L.P. Beria. He started forcing very strongly and in details nuclear investigations. Kapitza protested claiming that incompetent officer cannot in so active way instruct the scientists. Beria was stronger. Kapitza has been dismissed from the Committee, lost his position of the Director of the Institute, and was expelled from Moscow.

In 1972 the University of Wrocław, upon recommendation from Professor Włodzimierz Trzebiatowski, Director of the Institute of Low Temperatures and Structural Research of the Polish Academy of Sciences, awarded P. L. Kapitza with the title of Doctor Honoris Causa. When Kapitza arrived to receive the distinction, in his "doctor clarissimus" speech he highly praised and underlined the activity of Polish intelligentsia and lauded the ideas of freedom as an example inspiring the Russian intelligentsia. The Kapitza speech greatly irritated the Soviet consul from Poznań present at the ceremony.

After return from abroad Landau was in the period 1932-37, the Head of theoretical physics in several Institutions in Kharkov. There visited him Niels Bohr and Wolfgang Pauli. In 1937 some of Landau's collaborators were arrested. He escaped to Moscow, where Kapitza employed him as the Head of the Division of Theoretical Physics. At the end of April 1938 Landau prepared (according to official accusation) the project of the antibolshevik leaflet which should be distributed during the 1st May manifestation. There were among others the following sentences: the Stalinist clique made fascist revolution - socialism reminded only on the pages of lying newspapers - the only solution is the fight with the Stalinist and Hitler fascism.. "Signed: Moscow Committee of Antifascism Worker Party". Landau was arrested at April 28th 1938. P. Kapitza wrote immediately a letter to Stalin, comparing L.D. Landau to W.A. Fock, who was considered as the best Russian scientist. On April, the 6th, 1939, Kapitza wrote to W. Molotov (the top 
member of the authorities of the communistic party) since year for unknown reasons the Soviet, but also the World Science are deprived of the Landau skills. On April, the 26th, 1939 Kapitza wrote to Beria ("the bloody" soviet commissar): Dismiss please the arrested Professor of physics Lev Davidovich according to my personal guaranty. On September 23th 1938 Niels Bohr wrote to Stalin: I ask you kindly to consider the problems of Professor Landau... so that so highly talented scientist could come back to work so important for the progress of humanity. Fortunately, because of some unknown reasons, the interventions were fruitful and at April 28th 1939 Landau was free.

After imprisonment Landau's ambition was to create an ideal School of theoretical physics. His individual scientific work was extremely creative and devoted to the numerous quite different branches of physics. In addition essential meaning for success of the School had seminars of theoretical physics at the Institute of Physical Problems. Some inspiration to these Seminars was earlier participation of Landau in the seminars of Niels Bohr in Copenhagen. Similarity was in the very high scientific level. The differences were connected with an impulsive character of Landau.

Very often the speakers at the seminar were young people, students, aspirants (doctorants), for which the proper presentation of the papers was a chance to their further carrier. Usually Landau proposed them to present paper printed in Phys. Rev., which he considered interesting, according to his first filling. However, when Landau realized that the results were not deep, not interesting, the presentations were drastically interrupted. The most delicate procedure was when during the talk Landau asked the secretary of the seminar who is the next? Sometime the lecturer simulated that he does not understand the allusion. He tried very quickly to present next details with hope that they will be considered as more interesting. In such situation irritated Landau used very often world "won", i.e. be gone!

It is worth bring to mind that at that time Phys. Rev. issues were locked out in a special room and distributed only after personal Landau recommendation. Usually, after seminar, the group of the closely connected with Landau collaborators surrounded him and prolonged discussion. I joined them.

After some time I realized that the best understanding is when I speak with A.A. Abrikosov about superconductivity and with I. M. Khalatnikov about superfluidity. They introduced me to Lev Davidovich. Therefore, he knew, that I arrived to seminar from Dubna, I am from Poland, and that now I am interested in the theory of superconductivity. Once he informed me that he will organize the Conference devoted to quantum fluid and invited me. The place of the Conference was quite untypical, namely the industrial town Swierdlovsk, situated in the picturesque Ural mountains. Along this chain of mountains the border between Europe and Asia is traced. Every $1000 \mathrm{~km}$ there are beautiful obelisks marking the symbolic border line. There was organized excursion to the closest obelisk. I can boast that (thanks to Landau) my right leg was in Asia, and the left leg in Europe (it is documented on a photo).

As I mentioned before from the people of Landau School I had the most friendly relation with Aleksiej A. Abrikosov, for me Aljosha. He was born in 1928 in Moscow. Since 1948 he worked under Landau direction (after "theory minimum"). We both were interested in the problems of superconductivity, electrodynamics. Therefore, we had some common problems for discussions. Very often, we would continue them during dinner. From time to time after Seminar Aljosha invited me to special place, named very modestly the "Mess-hall" of the USSR Academy of Sciences. One can meet there very famous, prominent persons from "the World of Science". For these reasons this "hall" 
was specially supplied ( in known "socialistic manner") in exclusive food. I retained in memory, among others, lonely Lysenko. He was very aggressive therefore alone. Quite different was e.g. Igor Tamm, well known theoretical physicist always cheerful. Initially, Aljosha lived in Moscow close to the local "Savielov" train station. Just there I arrived from Dubna. Very often, I visited him early in the morning and after a good breakfast we went by car to the Landau Seminar. I really wanted to invite Aljosha to Poland. The best occasions were organized, since 1964, in Karpacz (Sudeten Mountains), the socalled Winter Schools in Theoretical Physics. Aljosha attended these meetings in 1974 and 1979 (together with A.J. Leggett). During longer walks we had time for discussions. Moreover, Aljosha was invited for several weeks to Wrocław as a guest of University and Institute of Low Temperature and Structural Research of the Polish Academy of Sciences (where I had also a permanent appointment). He arrived with his wife Ani, and they accepted invitation to stay in our home.

When I organized the Winter Schools of Theoretical Physics in Karpacz, I was of course interested to invite the most recognized foreign specialists. Among them Aljosha recommended to me Professor Charles P. Enz from Geneva University. He was W. Pauli graduate and then Ph.D. student. Under Pauli recommendation, Charles spent two years in Princeton (Institute for Advanced Study). In 1958, when Pauli was seriously ill, Charles had taken the special care of him in the hospital. Charles related that when Pauli was unexpectedly moved to another room, much more comfortable, he was really very frustrated. Charles tried to understand why? Pauli said: "look, what is the number of this room. It is 137 ! The deep understanding of the essence of this number (in reality $1 / 137=\alpha$, fine structure constant) during my whole life finished in failure". And Pauli died in the room number 137 (in 1958). According to Aljosha suggestion, I invited to the Karpacz Winter School (1970), which I organized Liquid Helium and Many Body Problems, participants, e.g. C.N. Yang, S. Peletminsky. Unfortunately, because of illness, Charles did not participate at this School (but he was later, namely in 1974 and in 1980). Therefore, I invited him in 1971 to our Institute of Theoretical Physics in Wrocław. As consequence, in the next year 1972 he invited me for longer stay in Geneva University with nomination of the President of the Genéve Canton for founctions de professeur invité. It was really very fortunate coincidence that in 1972 Ecole Politechnique in Lausanne invited Professor John Bardeen for three-month course of lectures about superconductivity: Fluctuations dans les superconducteurs. At that time it was 16 years after the publication of the microscopic theory of superconductivity proposed by Bardeen, Cooper, and Schrieffer, i.e. BCS theory. Bardeen presented the most important, interesting "fruits" of this theory. He arrived in the glory of Nobel Prize, which he got in 1956 together with W. Schockley and W.H. Brattain For their investigations of semiconductors and discovery of the transistor effect.

J. Bardeen was born in 1908. He studied, among others, in Princeton, and was employed at the Harvard University, next in the Bell Telephone Laboratories. Lately, at University of Illinois in Urbana. When me and Charles learned about J. Bardeen lectures (as I remember they took place twice per week) we attended them from Geneva with passion. Unfortunately, just after lecture, because of obligation at our University, we had to return to Geneva. During the usual breaks in the lectures, listeners went to a great terrace. Lausanne, like Geneva is situated on the brink of the very picturesque Lake Leman. Therefore, from the terrace there was a splendid view of the lake and the mountains. During breaks in lectures Professor Bardeen was permanently and compactly surrounded by participants of his lectures. I tried to speak with him many times, but 
when finally I succeeded to be closer to him, it was just the end of the break. Once, when at the Institute in Geneva was free day, we decided with Charles to remain in Lausanne for the whole day and find in the Library some interesting us literature, not available in Geneva. After several hours I decided to go to the cafeteria. By the way there were doors to terrace. Unexpectedly, I noticed Professor Bardeen quite alone, gazing at the beautiful panorama. Spontaneously, I accelerated, but slowed down immediately because my head was totally empty. How to start the conversation? I said finally that it is so nice combination of water and mountains, and when it is really good weather one can see ornament of these mountains... here I should say Mont Blanc, but I said: Mount Everest. Professor smiled and next said slowly and softly: It is too far. I understood immediately that the beginning of the conversation was not very intellectual. Charles, when I described this story cried of joy.

Fortunately Bardeen was really interested in conversation when he learned that I have permanent contacts with "the East World" of Science and I am situated in Dubna and Moscow. I related some interesting him details about Bogolyubov, Landau and members of his School, the Thursday Seminars, but also about the strong personality of Kapitza. Additional occasion to talk to him longer was a special reception at the end of Bardeen stay in Lausanne. I have obtained a pleasant dedication on his published lecture notes. During the stay in Lausanne, he gained a good reception. Therefore, at the end of year (1972) there was a very warm announcement that John Bardeen, together with L.N. Cooper and J.F. Schrieffer was awarded by the Nobel Prize: for their theory of superconductivity, usually called the BCS theory.

Since 1972 I visited University of Geneva almost every year. I visited also regularly Zürich, invited there by Professor Armin Thellung. He was, like Charles, asistant of W. Pauli. He attended "my" Karpacz Winter School in 1974. Armin was real admirer of classic music. Therefore, his obligation was to supply Pauli with tickets to all interesting concerts. I was invited to Zürich University to give seminars. On the other hand, I and Armin were interested in seminars of IBM Zürich Research Laboratory, where in the foreground was Professor K. Alex Müller, a known experimental physicist. He was strongly connected to Geneva, where he started to work after his studies in Zürich. Therefore, he visited very often Geneva, as a member of several Scientific Councils.

Karl Alexander Müller was born in Basel in 1927. He studied at Faculty of Physics and Mathematics ETH in Zürich. At the end of studies he attended the lectures by Wolfgang Pauli and was so impressed by his personality, that decided to become also a scientist. In 1958-63 A. Müller got leading position in the Batelle Memorial Institute in Geneva. Maybe for this reason, he visited often Geneva. Later on, he got position as research-staff member at IBM Zürich Research Laboratory. Since 1972 he was the leader of the group performing investigations. In 1982 he received the honorary status of IBM Fellow. Just after his studies he was interested in paramagnetic resonance investigations considering that his doctorate will be based on it. Really very fortunately somebody suggested him investigation of the quite new synthesized compound $\mathrm{SrTiO}_{3}$. Almost 15 years later Alex Müller was involved in investigations of the properties of this compound (and related perovskite compounds). He got the doctorate diploma in 1958. In 1964 it was discovered that "his" compound $\mathrm{SrTiO}_{3}$ is a superconductor, but with very low critical temperature, namely $T_{\mathrm{C}}=0.97 \mathrm{~K}$. The year 1964 was crucial in this respect that additionally the superconductivity has been discovered in oxide (i.e. nonmetallic) materials like $\mathrm{NbO}$ with $T_{\mathrm{C}}=1 \mathrm{~K}$ and $\mathrm{TiO}$ with $T_{\mathrm{C}}=2 \mathrm{~K}$, having still low $T_{\mathrm{C}}$. 
In 1982 two theoretical physicists: V.L. Ginzburg with O.A. Kirzhnitz published a paper devoted to the estimate of the possible maximal critical temperature $T_{\mathrm{C}}(\max )$ for metallic superconductors (metals and alloys). They obtained $T_{\mathrm{C}}(\max ) \approx 23 \mathrm{~K}$. As concerns the experimental results, the highest $T_{\mathrm{C}}$ were discovered only for niobium $(\mathrm{Nb})$ alloys. Namely, for NbGe we have $T_{\mathrm{C}}=23.2 \mathrm{~K}$ and for $\mathrm{NbSn} T_{\mathrm{C}}=18.05 \mathrm{~K}$. We see that till now the experimental investigations confirmed the Ginzburg-Kirzhnitz "rule". The essence of this rule is that the "metal" superconductivity is in reality the low $T_{\mathrm{C}}$ superconductivity. Hence, the dream to have superconductivity at "room" temperatures cannot be realized by use of metal materials. By lucky coincidence, in 1986 there appeared in Alex Müller Laboratory a very ambitious student J.G. Bednorz, in order to prepare there his diploma work. He was interested specially in properties of fashionable at that time compound $\mathrm{SrTiO}_{3}$. Therefore, he applied to the proper Master. Alex Müller immediately perceived his penetrability, intelligence, perseverance in scientific investigations. In the same year (1986) K.A. Müller and J.G. Bednorz published paper Possibility high $T_{\mathrm{C}}$ superconductivity in the Ba-La-Cu-O system (Z. Phys. B 64, 189 (1986)). It was the discovery of superconductivity in the nonmetal material with critical temperature $T_{\mathrm{C}}=30 \mathrm{~K}>T_{\mathrm{C}}(\max ) \approx 23 \mathrm{~K}$. This means the Ginzburg-Kirzhnitz limit was exceeded. In reality it was discovery of the high-temperature superconductivity (HTS). Nearly, one year later, the group of Professor C.W. Chu (USA) informed about new high $T_{\mathrm{C}}$ superconductor $\mathrm{Y}-\mathrm{Ba}-\mathrm{Cu}-\mathrm{O}$, having really impressive $T_{\mathrm{C}}=93 \mathrm{~K}$ (Phys. Rev. Lett. 58, 908 (1987)). K.A. Müller and J.G. Bednorz already in 1987 were awarded by Nobel Prize for their important breakthrough in the discovery of superconductivity in ceramic materials. It was astonishing that C.W. Chu was not incorporated to this Nobel "team". V.L. Ginzburg visited Wrocław namely the Institute of Low Temperatures, in October 1988, i.e. two years after discovery of HTS. He had a very interesting lecture devoted to this subject. I still have a very nice transparency "Crystal Structure $\mathrm{YBa}_{2} \mathrm{Cu}_{3} \mathrm{O}_{7-x}$ " from his lecture and the copy of his newest review paper from Uspekhi Fiz. Nauk, both with dedications. I used often that transparency at my lectures about HTS. Visiting Geneva after 1987 I met there several times, now the "Nobelist" K.A. Müller. Traditionally, the Dean of the Faculty in the case of so distinguished visitor invites about ten persons to the lunch in a good Restaurant. I was always in this company. Alex Müller recognized me at once. He was very interested in the development of the political situation in Poland. Usually he started off by asking What about Lech Walesa?

The jubilee year 1995, i.e. 50 years after the end of the II World War was closer and closer. One started with propositions of the special celebrations in Wrocław and territories joined to Poland 50 years ago. It was suggested that also the development of science, since 1945 in High Schools in Polish Wrocław should be included in the celebration. I was elected as Head of the Wrocław Branch of the Polish Physical Society for the period 1992-1996. I had a feeling that physicists should also join this "golden jubilee". Therefore, at XXXII Meeting of Polish Physicists in Kraków I gained acceptance that the next, XXXIII Meeting in 1995 will be organized in Wrocław. Fortunately, the same year there was also 75th Anniversary of the Polish Physical Society. The Head of the Organizing Committee was Professor Ewa Dobierzewska-Mozrzymas. As organizers of such all-Poland enterprise we realized that the rank of the Conference would be really high, when among the participants Nobel Prize Winners will be present. Fortunately, with my candidate Alex Müller from Zürich there was no problems. He accepted at once the invitation. By the way we realized that invitation of Nobel Prize Winner even one year in advance is not easy, because they have many obligations. The second honorary guest was 
Klaus von Klitzing from Stuttgart. He got Nobel Prize in 1989: for the discovery of the quantized Hall effect. The meeting in Wrocław took place in the period 18-21 September (1995). The time was adjusted to the very important International Conference: Recent Developments in High Temperature Superconductivity, the first Polish-US Conference (in Duszniki Springs, September 11-15). Both Conferences were organized just in this period of time, so Alex Müller could participate in both of them during a single visit to Poland. The meeting in Duszniki Springs (Sudeten Mountains) was organized by the Institute of Low Temperatures and Structure Research of the Polish Academy of Sciences, personally by Professor Jan Klamut. Interesting detail that initiator of this meeting was his son, Dr Peter Klamut. From the USA persons such as C.E. Chu, D. Pines, and V.J. Emery were present. Vic Emery, friend of mine, was an unknown specialist of quantum fluids and employed in the Brookhaven National Laboratory (on Long Island, close to the New York University at Stony Brook). I was invited several times to the New York University by C.N. Yang. When visiting this University I was "automatically" invited to Brookhaven Lab.

Alex Müller had very interesting lecture On the "s" and "d" Wave Symmetry in High- $T_{\mathrm{C}}$ Cuprate Superconductors. He changed the announced subject of presentation at "last minute" considering that the new one is specially interesting because of new recent experimental data. My lecture Superconducting Properties of the Weakly Interacting Charged Bose Gas presented results obtained together with Charles P. Enz. From Duszniki Springs we moved to Wrocław. The Center of the Conference was in Aula of the Wrocław Technical University. The cover of Pryzmat - Informal Journal of the Technical University introduced us to the atmosphere of the Congress. There we could ting in capital letters the following inscription:

$$
\begin{aligned}
& 75 \text { years of the Polish Physical Society } \\
& \text { XXXIII Congress of the Polish Physicists } \\
& 50 \text { years of Polish Science in Wroctaw }
\end{aligned}
$$

At the Wrocław Jubilee the Honorary Guests had following lectures: Karl Alex Müller: On the Development of the High Temperature Superconductivity, Klaus von Klitzing: From Microelectronics to Nanoelectronics. I was the initiator and the editor of the book Fizyka Wroctawska 1945-1995 (Physics in Wroctaw 1945-1995), elaborated by Heads of all Institutes of Physics in Wrocław. Every participant have received this publication.

In materials of the first Polish-US conference K.A. Müller printed only Summary of his paper in print in the Nature: Possible Coexistence of $s$-and $d$-Wave Condensate in Cooper Oxide Superconductors. (Nature 377 (1995)). I have received by letter a copy with dedication: To Professor Galasiewicz in remembering my stay in Wroctaw in September 1995, with best regards K. Alex Müller. Upon his suggestions, I published in 2000 the paper Superconducting Properties of the Charged Weakly Interacting Bose Gas (d Symmetry). It was the end of my active interest in the superconductivity.

\section{Memoirs}

[1] Robert Jungk, Heller als Tausend Sonnen, Stuttgart 1963 (Polish transl. 1967).

[2] Z.M. Galasiewicz, Superconductivity and Quantum Fluids, Oxford 1970.

[3] Z.M. Galasiewicz, My connections with prof. N.N. Bogoljubov in the Laboratory of Theoretical Physics in Dubna, J. Phys. Studies 2, 3 (1998).

[4] Z.M. Galasiewicz, Pioneers of Quantum Fluids - Honorary Doctors of Our University, Proc. 17th Max Born Symposium, Wrocław 2002. 
[5] H. Grynberg, Drohobycz, Drohobycz, Warsaw 1997 (in Polish) (informations about the Landau School).

[6] B. Weres, Soniatna Teorema (Sunny Theorem), Kiev 1975 - see p. 142, (in Ukrainian).

[7] W. Kusz, Nazbyt Bliskie Sasiedztwo - Kulisy Radzieckiej i Polskiej Atomistyki po II Wojnie Światowej (Too Near Neighborhood - Behind the Curtain of the Soviet Polish Atomic Physics after the II World War), Warsaw 1955 - see p. 52. 